\title{
Theoretical investigation of light absorption and scattering by nanoparticles
}

\author{
S.I. Pokutnyi ${ }^{1}$ and V.V. Kovalchuk ${ }^{2}$ \\ ${ }^{1}$ Educational and Research Center of the Odessa State University; Illichivsk 68001, Ukraine, vul. Danchenko, 17A; \\ Tel.: +0486843076 \\ ${ }^{2}$ South Ukrainian State University; Odessa 65045, Ukraine, vul.Staroportofrankovskaya, 26; Tel.: +0482 2435 70; E-mail. \\ wladik@tekom.odessa.ua
}

\begin{abstract}
In the framework of the dipole approximation we predict theoretically high magnitudes of: (1) oscillator strength transitions, and (2) the resonance light cattering $\left(\sigma_{s s}(\omega, a)\right)$ and absorption $\left(\sigma_{a b s}(\omega, a)\right)$ cross-sections. We suggest that these pecularities can be observed experimentally. We discuss the dependence of $\sigma_{a b s}(\omega, a)$ and $\left.\sigma_{s s}(\omega, a)\right)$ on light frequency $\omega$ and radius $a$ of the nanoparticle at different physical conditions.
\end{abstract}

Keywords: nanoparticles, absorption, scattering, spherical particles.

Paper received 26.11.99; revised manuscript recieved 14.12.99; accepted for publication 04.01.00.

\section{Introduction}

Optical properties of nano-dimensional structures (NDS) which form systems consisting of small semiconducting or dielectric spherical particles (SP) with diameter, being of the order of $1-10 \mathrm{~nm}$, distributed in the different material matrices [1-5] are a subject of intensive studies at the present time.

Large nonlinearity of the optical characteristics and short lifetimes of the photoexcited charged carriers in NDS allow us to treat such heterophase systems as prospective materials for the production of new optics and optoelectronic devices controlling, in particular, light signals.

The main task of this paper is to develop the theory of light absorption and scattering on the one-particle local states in the NDS. These are very interesting results for the studying the behaviour of NDS, which have a cluster structure [5-8].

Linear dimension of the semiconducting particles will be comparable with the linear dimension of the characteristic quasi-particles in semiconductors. Under this condition the effect of the spheric boundary between the two materials, having different dielectric constants (DC), can cause the sizequantization of carriers energy spectra due to both the pure boundedness in space of the quantization region [4] and to the polarization interaction of charge carriers (CC) with the semiconducting SP surface [9-11].
It was proved in [9-10] that under some definite conditions the localized one - particle states (LOPS) of CC of different kinds might appear. Such states are, for example, the localized states of charge carriers in semiconductors near semiconductor or dielectric particle, called the localized surface states [11-12], or the localization of $\mathrm{CC}$ inside of the small spherical volume, called the volume localized states. The surface localization may appear near an outer or inner spherical interface between the two dielectric media and are called the outer surface states [11,12] or the inner surface states [13], respectively.

At present there exists a sufficient amount of experimental data confirming the existance of LOPS's under consideration. For example, the localization of CC and excitons have been studied in the copper chloride [4], cadmium sulphate $[4,14]$ as well as SP in isoelectronic solid state solutions $\mathrm{A}_{2} \mathrm{~B}_{6}[15,16]$. In addition, the localization of the charge carriers on the antimony particle in $n$-type germanium and on the mercury particle placed in the dense mercury steam have been observed in [17] and [18], respectively.

\section{One-particle local states of charge carriers}

The problem of the CC localization near the spheric interface between two dielectric media has been analyzed in [9] using the following simple model of NDS: a SP with diameter $a$ and dielectric permittivity (DPr) $\varepsilon_{2}$ is surrounded by matrix with $\operatorname{DPr} \varepsilon_{1}$ and a quasiparticle with charge $e$ 


\section{S.I. Pokutnyi and V.V. Kovalchuk: Theoretical investigation of light absorption...}

moving either in the medium with DPr $\varepsilon_{1}$ and effective mass $m_{1}$ near the interface surface (the outer problem (OP)) or in the medium with DPr $\varepsilon_{2}$ and effective mass $m_{2}$ inside spherical volume (the inner problem (IP)). In addition, the polarization interaction $U(r, a)$ ( $r$ denotes the distance between a charge carrier and the SP center)with the surface charges induced on the spherical interface has been taken into account. We point out that the magnitude of $U(r, a)$ depends on the relative dielectric permittivity $\varepsilon=\varepsilon_{1} / \varepsilon_{2}$.

Let us write down the expression $U(r, a)$ for the interaction of charge carrier with the surface of SP [9] in the following forms: for the OP $(r>a)$

$$
U(r, a)=\frac{e^{2} \beta}{2 \varepsilon_{1} a}\left[\frac{a^{2}}{r^{2}-a^{2}}-\left(\frac{a}{r}\right)^{2} F\left(1, \alpha, \alpha+1 ;\left(\frac{a}{r}\right)^{2}\right)\right]
$$

for the IP $(r<a)$

$$
U(r, a)=\frac{e^{2} \beta}{2 \varepsilon_{2} a}\left[\frac{a^{2}}{a^{2}-r^{2}}-\frac{1-\alpha}{\alpha} F\left(1, \alpha, \alpha+1 ;\left(\frac{r}{a}\right)^{2}\right)\right](2)
$$

where $F\left(1, \alpha, \alpha+1 ; z^{2}\right)$ is the Gauss hypergeometric function, $\alpha=\varepsilon_{1} /\left(\varepsilon_{1}+\varepsilon_{2}\right)$ and $\beta=\left(\varepsilon_{1}-\varepsilon_{2}\right) /\left(\varepsilon_{1}+\varepsilon_{2}\right)$

There exist two possibilities for the charge carriers moving near the SP:

1. The polarization interaction $U(r, a)$ (cf. Eqs (1),(2)) leads to the attraction of $\mathrm{CC}$ by the SP surface and formation of the outer (for $\varepsilon<1[9,10]$ ) and inner (for $\varepsilon>1$ [11]) surface states;

2. For $e<1$ the polarization interaction (2) causes repulsion of CC from the inner surface of SP and formation of the volume localized states $[12,13]$ near the center of SP. This effect is called the volume localization.

We notice, that in [9-13] the potential $U(r, a)$ for IP has been obtained in the form of infinite series with respect to variable $(r / a)<1$. It has been shown in [9-13] that with decreasing of the SP diameter $a$ there appears the sizequantization effect reventing from $\mathrm{CC}$ localization because of the relative diminishing of the potential energy (1) or (2) in comparison with the kinetic energy. The smallest critical SP diameter $a_{c}$, for which the local state is emerging, is approximately equal to $b_{i}$ denoting the average distance of charge carrier localized in the ground state over the flat interface surface

$a_{c} \sim b_{i}=\frac{6}{|\beta|} a_{B_{i}}$

where $a_{B_{i}}=\varepsilon_{\mathrm{i}}\left(m_{0} / m_{i}\right)\left(\hbar^{2} / m_{0} e^{2}\right)$ is the Bohr diameter of $\mathrm{CC}$ in the medium with $\varepsilon_{i}(i=1,2) ; m_{i}$ and $m_{0}$ are the effective masses in the medium with $\varepsilon_{i}$ and the free carrier mass, respectively.

The energy spectrum of inner and outer surface states determined in $[5,9,11]$ employed the variational approach with the variational function in the form: for inner surface states $[5,11]$

$\chi_{l}\left(x, S_{2}\right)=A\left(S_{2}-x\right)^{l+1} x\left(2 S_{2}-x\right) \exp \left(-\gamma_{l}\left(S_{2}\right) x\right)$

and for outer surface states [5,9]

$$
\chi_{l}\left(x, S_{1}\right)=B x\left(S_{1}+x\right) \exp \left(-\mu_{l}\left(S_{1}\right) x\right)
$$

where $l$ denotes the orbital quantum number of $\mathrm{CC}, S_{i}=a / b_{i}$, $x$ is the CC distance from the SP surface in $b_{i}$ units (cf. Eq. (3)). The dependence of the variational parameters $\gamma_{l}\left(S_{2}\right)$ and $\mu_{l}\left(S_{1}\right)$ on the radius $S_{i}$ is depicted in Figs 1 and 2 , respectively, for $l=0,1$.

It has been shown in $[12,13]$ that the lowest-energy spectrum $E_{t, l}\left(S_{2}\right)$ of the CC volume local states in potential (2), for $\varepsilon<1$, is given by

$E_{t, l}\left(S_{2}\right)=\omega_{l}\left(S_{2}\right)(t+3 / 2)$

$\omega_{l}\left(S_{2}\right)=\frac{2 \sqrt{3}}{S_{2}^{3} / 2} \times$

$\times \sqrt{\frac{8 \beta}{3(1+\alpha)}+\frac{2(7+5 \alpha)}{2+\alpha} \sqrt{\frac{\beta L^{2}}{2 S_{2}(1+\alpha)}+\frac{\left(8+11 \alpha+5 \alpha^{2}\right) L^{2}}{2 S_{2}(2+\alpha)}}}$

where $t=2 n_{r}+l=0,1,2, \ldots$ is the principal quantum number, $n_{r}=0,1,2, \ldots$ is the radial quantum number,

$\omega_{l}\left(S_{2}\right)$ denotes the $\mathrm{CC}$ vibration frequency measured in

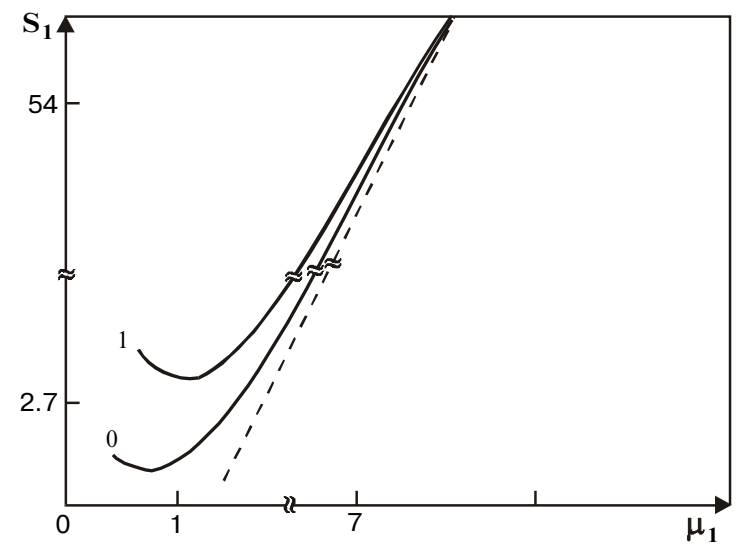

Fig. 1. Dependence of variational parameter $\mu_{1}\left(S_{1}\right)$ on the SP radius $S_{1}$. The curves labeled by 0 and 1 correspond to quantum number values $l=0$ and $l=1$, respectively. The dotted line corresponds to $S_{1}=(2 / 3) \mu$. 


\section{S.I. Pokutnyi and V.V. Kovalchuk: Theoretical investigation of light absorption...}

the $R_{y}=\frac{\hbar^{2}}{2 m_{2} a_{B_{2}}^{2}}$ units and $L_{y}^{2}=l(l+1)$.

In [17] the energy spectrum of CC localized near the interface of two different dielectric materials has been calculated theoretically under assumption that dielectric permittivities and potential $U(r, a)$ are continous at the interface. It can be shown that inclusion of noncontinuities of DPr's and potential $U(r, a)$ leads to the corrections of order of $\leq 7 \%$ in spectra $E_{t, l}\left(S_{2}\right)$ (for $\left.l=0,1\right)$ for all CC localized states [5,9-13] mentioned above.

\section{Dipole moments of the charge carrier transitions}

In the range of frequencies corresponding to the energies of the considered states of CC localized near spheric interface between two media, the electromagnetic wave length is much longer than the linear dimension of all local states which are of order of $b_{i}$ (cf. Eq.(3)). Therefore, their behaviour under electromagnetic field action can be well described in the framework of the dipole approximation. Moreover, operators of the dipole moments for the outer and inner problem have the following form [20]:

$$
\vec{D}(\overrightarrow{\mathbf{r}})=\left[1-\frac{\beta}{1+\alpha}\left(\frac{a}{r}\right)^{3}\right] e \overrightarrow{\mathbf{r}}
$$

and

$$
\vec{D}(\overrightarrow{\mathbf{r}})=\frac{3 \varepsilon_{1}}{2 \varepsilon_{1}+\varepsilon_{2}} e \overrightarrow{\mathbf{r}},
$$

respectively.
In order to estimate the magnitudes of the dipole moments for $\mathrm{CC}$ transitions induced by the external electromagnetic field we will study the transitions between lowlying local states of all considered types taking into account the parity of the corresponding states. For example, transitions between $s$ - and $p$-states are permitted for the dipole transitions, and changes $l \rightarrow l \pm 1$ of the orbital quantum number are accompanied with these transitions.

In order to calculate the matrix element of the dipole moment $D_{1,0}\left(S_{i}\right)$ for the charge carrier transition from $s$ to $p$-state, we assume that the monochromatic homogeneous electromagnetic wave $\varepsilon(\omega, t)$ propagates parallely to the OZ-axis, where $\omega$ denotes the light frequency. As the external perturbation, causing the studied dipole transitions, we take for the OP and IP the dipole moments defined by Eqs (8),(9) which are induced by the external electromagnetic field $\varepsilon(\omega, t)$. Finally, the matrix elements of the transition dipole moments from $s$ - to $p$-state of CC can be written in the form:

$$
\vec{D}_{1,0}\left(S_{i}\right)=\int_{0}^{2 \pi} d \phi \int_{0}^{\pi} \sin \theta d \theta \int_{\eta_{1}}^{r_{2}} r^{2} \psi_{1}\left(r, S_{i}\right) \vec{D}(r) \psi_{0}\left(r, S_{i}\right) d r,(9)
$$

where $\psi_{1}\left(r, S_{i}\right)$ and $\psi_{0}\left(r, S_{i}\right)$ are the wave functions of charge carrier in $s$ - and $p$-state, respectively; $\theta$ and $\phi$ denote the polar and azymuthal angles, respectively; $r_{1}=a, r_{2}=\infty$ for outer local states and $r_{1}=0, r_{2}=a$ for inner local states.

In the case of volume states such dipol transitions corresponds to the transition between the quantum states with $t=0(l=0)$ and $t=1(l=1)$. Using the formulae for wave functions of quantum oscillator and taking into account Eqs (6),(8) and (9) we can obtain the magnitude of the matrix elements of dipole transition moments $D_{1,0}$ between states with $t=0$ and $t=1$ localized in the SP center [21].

$$
D_{1,0}=\frac{S_{2}^{3 / 4} e b_{2}\left(3 \varepsilon_{1} /\left(2 \varepsilon_{1}+\varepsilon_{2}\right)\right)}{3^{7 / 6} 2^{3 / 2}\left[\frac{8 \beta}{3(1+\alpha)}+\frac{2(7+5 \alpha)}{2+\alpha} \sqrt{\frac{\beta}{S_{2}(1+\alpha)}}+\frac{8+11 \alpha+5 \alpha^{2}}{S_{2}(2+\alpha)}\right]^{1 / 4}}
$$

In order to estimate magnitudes of the matrix elements of dipole transitions corresponding to the inner $D_{0,1}\left(S_{2}\right)$ and outer $D_{0,1}\left(S_{1}\right)$ surface states we will use the variational wavefunctions (4) and (5), respectively. Taking into account the formulae (4), (8) and (5), (7) we can get the matrix elements of dipole transitions (9) between the ground state with $l=0$ and the first excited states with $l=1$ :

for inner surface states [23]

$$
\begin{aligned}
& D_{1,0}\left(S_{2}\right)=\frac{L_{2} S_{2} e b_{2}}{3 \sqrt{2}} \\
& L_{2}=2^{5} \frac{\sqrt{3} \varepsilon_{1}}{\left(2 \varepsilon_{1}+\varepsilon_{2}\right)} \frac{\tilde{\mu}_{0}^{7 / 2} \ddot{\mu}_{1}^{9 / 2}}{\left(\tilde{\mu}_{0}+\tilde{\mu}_{1}\right)^{9}} L_{2}^{(1)} L_{2}^{(2)} L_{2}^{(3)}
\end{aligned}
$$


where

$L_{2}^{(1)}=\left(\tilde{\mu}_{0}+\tilde{\mu}_{1}\right)^{5}-16\left(\tilde{\mu}_{0}+\tilde{\mu}_{1}\right)^{4}+125\left(\tilde{\mu}_{0}+\tilde{\mu}_{1}\right)^{3}-570\left(\tilde{\mu}_{0}+\tilde{\mu}_{1}\right)^{2}+1470\left(\tilde{\mu}_{0}+\tilde{\mu}_{1}\right)-1680$,

$L_{2}^{(2)}=\frac{1}{\sqrt{8 \tilde{\mu}_{0}^{4}-36 \tilde{\mu}_{0}^{3}+78 \tilde{\mu}_{0}^{2}-90 \tilde{\mu}_{0}+45}}$,

$L_{2}^{(3)}=\frac{1}{\sqrt{4 \mu_{1}^{6}-30 \mu_{1}^{5}+123 \mu_{1}^{4}-330 \mu_{1}^{3}+585 \mu_{1}^{2}-630 \mu_{1}+315}}$,

$\tilde{\mu}_{0}=\gamma_{0}\left(S_{2}\right) S_{2}$,

$\mu_{1}=\gamma_{1}\left(S_{2}\right) S_{2}$

and for outer surface states

$D_{1,0}\left(S_{1}\right)=2^{-1 / 2} L_{1} S_{1} e b_{1}$,

$L_{1}=2^{5 / 2} \frac{\left(\mu_{0} \mu_{1}\right)^{5 / 2}}{\left(\mu_{0}+\mu_{1}\right)^{6}} \frac{\varepsilon_{2}-\varepsilon_{1}}{\left(2 \varepsilon_{1}+\varepsilon_{2}\right)} \frac{3\left(\mu_{0}+\mu_{1}\right)^{2}+12\left(\mu_{0}+\mu_{1}\right)+20}{\sqrt{3\left(\mu_{0}^{2}+3 \mu_{0}+3\right)\left(\mu_{1}^{2}+3 \mu_{1}+3\right)}}$

where

$\mu_{0}=\gamma_{0}\left(S_{1}\right) S_{1}$,

$\mu_{1}=\gamma_{1}\left(S_{1}\right) S_{1}$.

and $j_{0}(x), j_{1}(x)$ are the Bessel spherical functions.

The variational parameters $\mu_{0}\left(S_{1}\right), \mu_{1}\left(S_{1}\right), \gamma_{0}\left(S_{2}\right), \gamma_{1}\left(S_{2}\right)$ occurring in the trial wave functions of the inner and outer surface localized states are determined [7,9].

We point out that numerical figures occurring in Eqs (10)-(12) result in calculations of dipole moments transitions [11] using variational or oscillator-type wave [22] functions for inner [4] and outer [5] surface states or volume [21] local states, respectively.

Let us note that Eq. (11), defining the dipole transition moment $D_{1,0}\left(S_{2}\right)$ for the inner surface states, is valid provided both inequalities $\exp \left(-\gamma_{1}\left(S_{2}\right)\right)<<1$ and $\exp \left(-\gamma_{0}\left(S_{2}\right)<<1\right.$ are satisfied. These conditions are fulfilled for arbitrary values of the variational parameters $\gamma_{0}\left(S_{2}\right)$ and $\gamma_{1}\left(S_{2}\right)$ (cf. Fig. 2) and for all magnitudes of the SP radii $S_{2}>S_{c}$.

As the example, let us write down the magnitudes of the dipole transition moments $D_{1,0}\left(S_{1}\right)$ (cf. Eq. (12)) for the outer problem and $D_{1,0}\left(S_{2}\right)$ (cf. Eq. (11)) for the inner problem in SP with radius $S_{1}=S_{2}=S=4[21]$ :

$D_{1,0}\left(S_{1}\right)=7.6 \frac{\varepsilon_{2}-\varepsilon_{1}}{2 \varepsilon_{1}+\varepsilon_{2}} e b_{1}$,

$D_{1,0}\left(S_{2}\right)=4.2 \frac{\varepsilon_{1}}{2 \varepsilon_{1}+\varepsilon_{2}} e b_{2}$.

The magnitudes of $D_{1,0}\left(S_{1}\right)$ and $D_{1,0}\left(S_{2}\right)$ given by Eqs (13) and (14) are practically constant with increasing SP radius and increase slightly at $S \rightarrow \infty$. This property follows from the fact that radius $a$ of the dipole has the linear dimension of the order of $b_{i}$ radius state, which practically does not vary.

We should note that due to this property the variational functions (4) and (5) used for the estimation of the matrix element magnitudes of the transitions moments (cf. Eqs (10)-(15)) do not lead to a sufficient error and have an effect on the numeric prefactor being of the order of $\sim 1$.

\section{Light absorption and scattering}

The results obtained so far and concerning the dipole moments of transitions for the volume states $D_{1,0}$ (cf. Eq. (10)) for the inner and outer states given by Eqs (11) and (12),

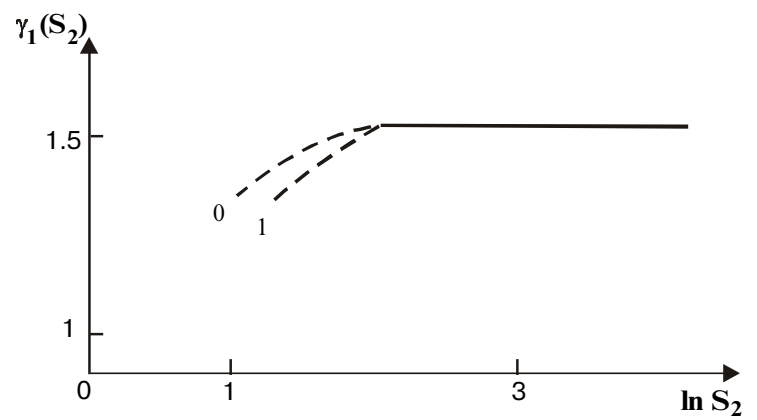

Fig. 2. Variational parameter $\gamma_{1}\left(S_{2}\right)$ versus $\ln \left(S_{2}\right)$, where $S_{2}$ denotes the SP radius $S_{2}$; the depicted numbers specify magnitudes of the quantum number $l$. 


\section{S.I. Pokutnyi and V.V. Kovalchuk: Theoretical investigation of light absorption...}

respectively, allow us to derive the light absorption and scattering in the considered NDS in the electromagnetic waves frequency range corresponding to the energies of LOPS's discussed in the previous section.

The absorption cross-section $\sigma_{a b s}(\omega, a)$ of the SP with radius $a$ is related to its polarization $A^{\prime \prime}(\omega, a)[22]$ in the form:

$\sigma_{a b s}(\omega, a)=\frac{4 \pi}{c} \omega A^{\prime \prime}(\omega, a)$,

where $c$ is light velocity in vacuum.

If the states of the charge carrier in SP are quantized, then their polarization $A^{\prime \prime}(\omega, a)$ can be easily found if we assume that the SP forms a single giant ion. In this case the polarization $A(\omega, a)$ of the charged SP can be expressed by the matrix elements of dipole transition moments between quantum states [22,23]. For sufficiently low temperatures fulfilling the inequality $T<E_{B}=\left(\hbar^{2} /\left(2 m_{i} a_{B}^{2}\right)\right)$, which are of the order of $1-10 \mathrm{~K}$ at $a_{B} \sim 10-10^{2} \AA$, i.e. for temperatures less then the binding energy $E_{B}$ of the considered states, we can write down the explicit form of the polarization

$$
A^{\prime \prime}(\omega, a)=\frac{e^{2}}{m_{i}} \sum_{j} \frac{f_{0 j}(a)}{\omega_{j}^{2}(a)-\omega^{2}-i \omega \Gamma_{j}(a)},
$$

where

$$
f_{0 j}(a)=\frac{2 m_{i}}{\hbar e^{2}}\left(\omega_{j}(a)-\omega_{0}(a)\right)\left|D_{j 0}(a)\right|^{2}
$$

is the oscillator strength of the charge carrier with effective mass $m_{i}$ transition from the ground to the $j$-th quantum state, $\omega_{0}$ is the energy of the ground state of the charge carrier, $D_{j 0}(a)$ denotes the matrix element of the dipole transition moment, $\hbar \omega_{i}(a)$ is the energy of the $j$-th quantum state and $\Gamma_{j}(a)$ means the width of the $j$-th excited state $[10,12]$.

If the bound states are absent, then $D_{j 0}(a) / e$ will be proportional to the linear dimension $a$ of the delocalization range of the charge carrier (for large $j$ we have $D_{j 0}(a)=$ $=a \cdot e / j[22])$. Moreover, the oscillator strength $f_{0 j}(a) \sim$ $\sim\left|D_{j 0} / e\right|^{2} \sim a^{2}$. In this case polarization $A^{\prime \prime}(\omega, a)$ (cf. Eq. (16)) and the absorption cross-section (15) for SP with radius $a$ have the following forms:
$A^{\prime \prime}(\omega, a)=\omega \cdot f(\omega) a^{2}$

$\sigma_{a b s}(\omega, a)=\frac{4 \pi}{c} \omega^{2} \cdot f(\omega) a^{2}$

since $f(\omega)$ does not depend on $a$, and $f(\omega)$ is weakly varied with $\omega[21]\}$ :

$\sigma_{a b s}(\omega, a) \sim \omega^{2} a^{2}$

If the bound states of $\mathrm{CC}$ were formed near the interface between two different media, then the main contribution to $A^{\prime \prime}(\omega, a)$, for $T<E_{B}$, would come from the transitions between quantum states of a discrete spectrum. The contribution of a single resonance state into (16) can be written in the form

$A^{\prime \prime}(\omega, a)=f_{01}(a) \cdot F\left(\omega_{1}, \omega\right)$,

where

$F\left(\omega_{1}, \omega\right)=\frac{e^{2}}{m_{i}} \cdot \frac{1}{\omega_{1}^{2}-\omega^{2}-i \omega \Gamma_{1}(a)}$

has the usual resonance form and in the vicinity of the resonance it is independent of $a$ [23]. Let us note that we have isolated single resonance contribution corresponding to the transition between the ground $s$ - and $p$-states discussed above for which $f_{01}(a)$ is defined by Eqs (17) and (10) $\div(12)$.

It is evident that a restriction of our studies to the consideration of the $s$ - and $p$-states does not influence on the obtained behaviour of $A^{\prime \prime}(\omega, a)$ and $\sigma_{a b s}(\omega, a)$ as the function of the SP radius $a$ since the dependence of the oscillator strength $f_{01}(a)$ has the same character for other states (cf. Eq. (17)).

From Eqs (15), (20) and (21) it follows that the resonance light

$$
\sigma_{a b s}\left(\omega, S_{i}\right)=4 \pi \frac{\omega}{c} f_{01}\left(S_{i}\right) F\left(\omega_{1}, \omega\right)
$$




\section{S.I. Pokutnyi and V.V. Kovalchuk: Theoretical investigation of light absorption...}

where the oscillator strength $f_{01}\left(S_{i}\right)$, in accordance with Eqs (10) and (17), for the volume states localized in the $\mathrm{SP}$ center with radius $S_{i}$ takes the form Eq. (23).

The transition oscillator strength $f_{01}\left(S_{i}\right)$ exhibits, for the surface and the inner states, the same dependence on SP radius $S_{i}$ and is given by

$$
f_{0 i}\left(S_{i}\right)=\frac{m_{i} b_{i}^{2}}{\hbar} \cdot S_{i}^{2} L_{i}^{2} \omega_{i}
$$

where the parameters $L_{i}$ for inner $(i=2)$ and outer $(i=1)$ surface states are defined by Eqs (11) and (12), respectively.

We point out that the comparison of Eqs (22) $\div(24)$ exhibits the different dependence character for the light $\mathrm{ab}$ sorption cross-section $\sigma_{a b s}(\omega, a)$ on frequency $\omega$ and radius $a$ of the dielectric particle, under different physical conditions.

If the bound states near the spheric interface between two media do not exist, then, in accordance with Eq. (19), we have

$\sigma_{a b s}(\omega, a) \sim \omega^{2} a^{2}$

In the case of the volume states of $\mathrm{CC}$ the absorption cross-section $s_{a b s}(\omega, a)$ is defined by Eqs (22), (23) and

$\sigma_{a b s}(\omega, a) \sim F\left(\omega_{1}, \omega\right) \cdot \omega \cdot a^{3 / 2}$

For the surface inner and outer states the absorption cross-section $\sigma_{a b s}(\omega, a)$, in accordance with (22) and (24), has the same dependence on SP radius $a$ : $\sigma_{a b s}(\omega, a) \sim F\left(\omega_{1}, \omega\right) \cdot \omega \cdot a^{2}$.

Thus, the localization of $\mathrm{CC}$ on the spheric interface and inside the NDS leads to qualitatively different dependence of the absorption of electromagnetic field on radius SP $a$ and light frequency $\omega$. This fact gives an additional experimental possibility to spectroscopic findings and studies of the considered localized states of charge carriers.

A similar possibility also gives the elastic scattering of electromagnetic waves with frequency $\omega$ on the SP. The scattering cross-section $\sigma_{s c}(\omega, a)$ is given by [22]

$\sigma_{s c}(\omega, a)=2^{7} \cdot 3^{-3} \cdot \pi^{3}\left|A^{\prime \prime}(\omega, a)\right|^{2} \cdot\left(\frac{\omega}{c}\right)^{4}$

and, according to Eqs $(20) \div(24)$ and $(18), \sigma_{s c}(\omega, a)$ exhibits different dependences on the radius $a$ and frequency $\omega$ for various types of considered localized states.

In fact, for inner and outer surface states, according to Eqs (20), (21), (24), the cross-section $\sigma_{s c}(\omega, a)$ of the elastic scattering of electromagnetic waves is given by the formula

$$
\sigma_{s c}(\omega, a)=2^{7} \cdot 3^{-1} \cdot \pi^{3} \cdot\left(\frac{\omega}{c}\right)^{4} \times
$$

$\times F^{2}\left(\omega_{1}, \omega\right) \cdot L_{i}^{2} \cdot\left(\frac{m_{i} b_{i}^{2} \omega_{1}}{\hbar}\right)^{2} \cdot S_{i}^{4}$.

The scattering cross-section $\sigma_{s c}\left(\omega, S_{2}\right)$, for the volume local states, according to Eqs (20), (21) and (23), has the following form:

$\sigma_{s c}\left(\omega, S_{2}\right)=\frac{2^{3} \cdot 3^{-5 / 3} \cdot \pi^{3} \cdot\left(\frac{\omega}{c}\right)^{4} F^{2}\left(\omega_{1}, \omega\right) \cdot\left(\frac{\varepsilon_{1}}{2 \varepsilon_{1}+\varepsilon_{2}}\right)^{4} \cdot S_{2}^{3} \cdot\left(\frac{m_{i} b_{i}^{2} \omega_{1}}{\hbar}\right)^{2}}{\frac{8 \beta}{3(1+\alpha)}+\frac{2(7+5 \alpha)}{2+\alpha} \cdot \sqrt{\frac{\beta}{S_{2}(1+\alpha)}}+\frac{8+11 \alpha+5 \alpha^{2}}{S_{2}(2+\alpha)}}$

If the bound states near the SP do not occur, then, according to Eq. (18), the dependence of the cross-section $\sigma_{s c}(\omega$, $S_{\mathrm{i}}$ ) (cf. Eq. (27)) on $\omega$ and $S_{i}$ is given by

$\sigma_{s c}\left(\omega, S_{i}\right)=2^{7} \cdot 3^{-1} \cdot \pi^{3} \cdot\left(\frac{b_{i}}{c}\right)^{4} \omega^{6} \cdot f^{2}(\omega) \cdot S_{i}^{4}$

The optical coefficient of attenuation $\eta(\omega, a)$, which takes into account the light absorption and scattering effects on the considered LOPS's near the small dielectric particles with radius $a$ and concentration $N$, may be written in the form

$\eta(\omega, a)=N\left[\sigma_{a b s}(\omega, a)+\sigma_{s c}(\omega, a)\right]$ 


\section{S.I. Pokutnyi and V.V. Kovalchuk: Theoretical investigation of light absorption...}

The last expression may be used for a group of noninteracting NDS. This condition is fulfilled if the inequality

$a_{c}<\frac{1}{N^{1 / 3}}$

holds true, where $a_{c} \sim b_{i}$ (cf. also Eq. (3)) denotes the SP's radius. The condition (32) is fulfilled for concentration not exceeding $N \leq 10^{14} \mathrm{~cm}^{-3}$ of small cadmium sulphate or cadmium selenite particles with $a_{c} \sim b_{i} \sim 10^{2} \AA$ (cf. [9]-[13] as documented in experimental studies $[1,2,14]$.

\section{Comparison of the theory with experimental data}

Finally, we briefly discuss physical situations for which the results obtained in this paper are of the particular importance. An interesting feature of the studied states of charge carriers localized on the spheric interface is removing of degeneracy with respect to the orbital quantum number $l$. As a result the distance between the energy sublevels will be sufficiently small [7-11].

If consider that: (A) The dipole moments of transitions for all the considered local states, according to Eqs (10)-(12), have the large magnitudes of $D_{1,0}(a) \sim e \cdot b_{i}>>1 D$ (i.e. $D_{1,0}(a)$ exceeds typical magnitudes for the transition dipole moments of the nonorganic semiconducting materials for which $D_{1,0}(a) \sim$ $10^{-1} D$ [24]; (B) The allowed dipole transitions induced by the electromagnetic field between the nearest energy sublevels correspond to a changhe in the quantum number $l$ by \pm 1 , then it is evident that the discussed NDS are strong non-linear media for the electromagnetic radiation. The last circumstance represents a special interest for construction of novel nonlinear devices in a wide wavelength range with its magnitude depending on the nature of the contacting materials.

As an axample, let us consider a nano-dimensional system which consists of the spherical semiconducting parti- cle of CdS with radius $a \cong 54 \AA$ placed in the boric-silicate glass. In this system it was found experimentally the oscillator-like energy spectrum for the hole states localized near the particle centre with the energies of $\sim 10^{2} \mathrm{meV}$ [25]. In [26],[27] it was shown that the spectrum of the hole volume local states studied experimentally in [25] consists of a series of equidistant energy levels, and the distance between these energy sublevels is proportional to $a^{-3 / 2}$. The dipole moments of hole transitions between such equidistant sublevels, according to Eq. (10), take high values, i.e. $D_{1,0} \sim 10 \div 20 D$.

Let us carry out a qualitative estimation of the crosssection $\sigma_{a b s}(\omega, a)$ and $\sigma_{s c}(\omega, a)$ of light on the above mentioned local states of $\mathrm{CC}$ for the isolated transition $|0>\rightarrow| 1>$ under the experimental conditions of [1]:[4],[23]. Let us assume that the light frequency $\omega$ is not comparable with the resonance frequency $\omega_{1}$ of the local state in the SP and let us suppose also that the broadening $\Gamma_{1}$ of the energy level $E_{1}=\hbar \omega_{1}$ is sufficiently small, i.e. $\left(\Gamma_{1} / \omega_{1}\right)<<1$. Then we can estimate the magnitudes of the cross-sections $\sigma_{a b s}(\omega$, $a$ ) and $\sigma_{s c}(\omega, a)$ using Eqs (15), (23), (24) and (27) for which the SP polarization takes the form

$A^{\prime \prime}(a)=\frac{e^{2}}{m_{i}} \cdot \frac{f_{01}(a)}{\omega_{1}^{2}}$.

In Table I the estimated magnitudes of: the oscillator strength $f_{01}(a)$ (cf. Eqs (23) and (24)), the polarization $A^{\prime \prime}(\omega$, a) (cf. Eq. (33)), the light absorption cross-sections $\sigma_{a b s}(\omega$, a) (cf. Eq.(15)) and $\sigma_{s c}(\omega, a)$ (cf. Eq. (27)) corresponding to the specified above one-particle localized states are listed for NDS containing the semiconducting (dielectric) matrix and dispersed in it the semiconducting (or dielectric) SP with radius $a \geq a_{c}$ [7-11].

From the presented estimations it follows that the crosssection $\sigma_{a b s}(\omega, a)$ takes super high magnitudes of $\sigma_{a b s}(\omega$, $a) \approx 1.3 \cdot 10^{-16} \mathrm{~cm}^{2}$ for light absorption on the hole volume local states in the semiconducting GaAs particle embedded in boric-silicate glass matrix (or in another medium with

Table I. Parameters of electron and hole bound states localized on nano-dimensional structures, made from materials specified in the second column, with dielectric permittivity $\varepsilon_{2}$ (cf. the third column) placed in dielectric materials with dielectric permittivity $\varepsilon_{1} ; a$ - the radius of $\mathrm{SP}, m_{h}$ - the effective hole mass in SP, $E_{1}$ - the electron or hole bound energy, $f_{01}$ - the oscillator strength transition, $A^{\prime \prime}$ - the polarization of SP, $\sigma_{a b s}$ and $\sigma_{s c}$ - the cross-section of light absorption and scattering, respectively.

\begin{tabular}{llllllllll}
\hline \hline$\varepsilon_{1}$ & $S P$ & $\begin{array}{l}a(\AA) \\
(\mathrm{S})\end{array}$ & $\varepsilon_{2}$ & $m_{h} / m_{0}$ & $\begin{array}{l}E_{1} \\
(\mathrm{meV})\end{array}$ & $\begin{array}{l}f_{01} \\
\left(10^{-1}\right)\end{array}$ & $\begin{array}{l}A^{\prime \prime} \\
\left(10-24 \mathrm{~cm}^{3}\right)\end{array}$ & $\begin{array}{l}\sigma_{a b s} \\
\left(10^{-24} \mathrm{~cm}^{2}\right)\end{array}$ & $\begin{array}{l}\sigma_{s c} \\
\left(10^{-24} \mathrm{~cm}^{2}\right)\end{array}$ \\
\hline 1.5 & GaAs & $\begin{array}{l}340 \\
(4)\end{array}$ & 13.2 & 0.62 & 50 & 3.56 & $2.6 \cdot 10^{2}$ & $1.3 \cdot 10^{8}$ & $2.4 \cdot 10^{-3}$ \\
1.5 & $\mathrm{CdS}$ & $\begin{array}{l}54 \\
(12.65)\end{array}$ & 9.3 & 5 & 50 & 0.18 & $1.6 \cdot 10^{2}$ & $4 \cdot 10^{4}$ & $6 \cdot 10^{-13}$ \\
1 & $\mathrm{He}$ & $\begin{array}{l}420 \\
(4)\end{array}$ & 1.06 & & 0.65 & 3.81 & $4.8 \cdot 10^{3}$ & $1.6 \cdot 10^{4}$ & $1.6 \cdot 10^{-17}$ \\
\hline \hline
\end{tabular}




\section{S.I. Pokutnyi and V.V. Kovalchuk: Theoretical investigation of light absorption...}

$\left.\varepsilon_{1}<<\varepsilon_{2}\right)$. We point out that the magnitude of $\sigma_{a b s}(\omega, a) \approx$ $1.3 \cdot 10^{-16} \mathrm{~cm}^{2}$ is $10^{8}$ times larger than the typical atomic values of $\sigma_{a b s}(\omega, a) \sim 10^{-24} \mathrm{~cm}^{2}$ [24].

The observed values of the cross-section $\sigma_{a b s}(\omega, a)$ take $\sim 10^{-20} \mathrm{~cm}^{2}$ for the light absorption by the inner surface states of the electron in the bublon (a macroscopic spherical cavity in a liquid superfluid) embedded in superfluid helium [30] as well as by the hole volume local states in the cadmium sulphate SP placed in boric-silicate glass matrix $[25,28]$. In addition, the elastic light scattering cross-section $\sigma_{s c}$ by the mentioned one-particle local states will be sufficiently small in comparison with $\sigma_{a b s}$, i.e. $\left(\sigma_{s c} / \sigma_{a b s}\right) \sim$ $10^{-11}$.

This leads to the conclusion that the light attenuation coefficient $\eta(\omega, a)$ (cf. Eq. (31)) is determined mainly by processes of light absorption by one-particle local states of charge carriers. The magnitude of $\eta(\omega, a)$, corresponding to light absorption by local states of NDS described in Table I, takes the values of $\eta(\omega, a) \sim\left(10^{-2} \div 10^{-6}\right) \mathrm{cm}^{-1}$ for the SP with radius $a>a_{c}$ (cf.Eq.(3)) fulfilling condition (32) for concentration of $N=10^{14} \mathrm{~cm}^{-3}$.

In this way the large absorption cross-section $\sigma_{a b s}$ of the electromagnetic field by one-particle local states of charge carriers occurring in the vicinity of the semiconducting (dielectric) particle in NDS offers an interesting possibility of applying such heterophase structures to new materials which strong electromagnetic waves absorption (in particular: the light) in a wide wavelength range with its magnitude depending on the nature of the materials in contact.

\section{References}

1. A. D. Yoffe,Low-dimensional systems:quantum size effect and electronic properties of semiconductor microcrystallites (zero-dimensional system) and some quasi-two-dimensional systems // Adv. Phys., 42(2), pp. 173-266, (1993).

2. L. Rossetti and L. E. Brus, Quantum size effects in the redox potential // J. Chem. Phys., 79(2), pp. 1086-1089, (1983)

3. M. Kulish, V. Kunets and M. Lisitsa, Optical methods of determination of parameters for nanocrystals in quasi-zero-dimensional structures // Ukrainskii fizicheskii zhournal, 41(11-12), pp.1075-1082, (1996).

4. A. Ekimov, A.A. Onushchenko and A. Efros, Quantization of an energy distribution of electron defects in an isentropic potential of an electron // Pisma v ZhETF, 43(6), pp. 337-339, (1986) (in Russian).

5. S. I. Pokutnyi and V.V. Kovalchuk, The spectrum of an exciton in nanocrystal semiconductor structures - theory, Chap. 3.15 in Metal clusters in chemistry, Eds. P.Braunstein, L.A.Oro,P.R.Raithby, Vol. 3, pp.1263-1270, WILEY-VCH, (1999).

6. V.V. Kovalchuk, The cluster modeling of the reaction on the $\mathrm{Si}$ surface, in Abstracts: 9th International Symposium on Small Particles and Inorganic Clusters. Lausanne, Switzerland, 1-5 September. P. 10.32, (1998).

7. V.V. Kovalchuk, L.Yu.Kutsenko et.al., Cluster Model in Surface Science in Computer modelling of electronic and atomic processes in solids. NATO Scientific Affairs Division, Eds. R.Tennison, A.Kiv, pp.63-74, Kluver Acad. Publ. DordrechtBoston-London, (1996).
8. The Chemistry of organic silicon compounds, Chap. 1, Vol.2(1), Eds. Z.Rapport, Y.Apelog, John Wiley-Sons, Chichester-N.Y.-Weinheim-Brisbane-Singapore-Toronto, (1998).

9. N.A. Efremov and S.I. Pokutnyi, Spectra of local states of charge carriers in dispersion mediums // Sov. Phys. Solid State, 32(10), pp.2921-2930, (1990).

10. S.I. Pokutnyi and N.A. Efremov, Broadening of quasi-stationary states of charge carriers in dispersion mediums// Phys.Tverd.Tela, 32(10), pp.2845-2851, (1991) (in Russian).

11. N.A. Efremov and S.I. Pokutnyi, Theory of macroscopic local single-particle charge ststes in quasi-zero-dimensional structures: Surface local states // Phys.Status Solidi, B165, pp.109-118, (1991).

12. S.I. Pokutnyi and N.A.Efremov, Theory of macroscopic local single-particle charge states in quasi-zero-dimensional structures: Bulk local states // Phys.Stat.Sol., B173(2), pp.573-582, (1992).

13. S.I. Pokutnyi, Sized quantization of charge carriers in quasizero structures // Phys.Tverd.Tela, 35(2), pp.257-264, (1993) (in Russian).

14. V. Grabovskis and A. Ekimov, Photo-ionization of semiconducting microchips in glass // Phys.Tverd.Tela, 31(1), pp. 272-275, (1989) (in Russian).

15. S. Permogorov and A. Reznitsky, Role of localization of excitons luminiscence of hard solutions $\mathrm{A}_{2} \mathrm{~B}_{6} / /$ Dokl. AN USSR, 49(10), pp. 2019-2025, (1985) (in Russian).

16. A. Reznitsky, S. Permogorov and A.Yu. Naumov, Localization of carriers of excitons visible electrons of hard solutions $\mathrm{A}_{2} \mathrm{~B}_{6} / /$ Dokl. AN USSR, 52(4), pp.691-696, (1988) (in Russian).

17. V. Shakhovtsov, S. Shakhovtsova and I. Yaskovets,Mobility of carriers of current in semiconductors with areas of a disordering // Fiz. Tekh. Poluprovodnikov, A 11(10). pp. 1967-1971, (1977) (in Russian).

18. P. Krebs and V. Girand, Electron localization density amony vapoure // Phys.Rev. Lett., 44(3), pp.211-213, (1980).

19. A. Gabovich, L. Ilchenko and E. Pashitsky, Electrostatic interaction of charges with a surface of semiconductors // Fiz. Tverd.Tela, 21(6), pp.1683-1689, (1979) (in Russian).

20. L.D. Landau and E.M. Lifshitz, Electrodynamics of Continuous Media, Pergamon Press, Oxford, (1984).

21. N.A. Efremov and S.I. Pokutnyi, Macroscopic local charge states in ultradispersion media. Preprint No 1 , Institute of spectroscopy, USSR Academy of Sciences, Moscow (1984).

22. S.I. Pokutnyi, The theory of absorbing and scattering of light in quasi-zero-dimensional structures: 1 . The dipole moments of charge carrier transitions // Fiz. Tverd. Tela, 39(4), pp. 702722, (1997) (in Russian).

23. V.M. Agranovich, Theory of Excitons, Pergamon Press, Oxford, (1970).

24. V.M. Agranovich and V.L. Ginzburg, Crystal Optics with Spatial Dispersion and Excitons, Springer Verlag, New York, (1979).

25. Yu. Petrov, Fizika malykh chastits, Nauka, Moskva, (1982) (in Russian).

26. N.W. Ashcroft and N.D. Mermin, Solid State Physics, New York, Holt, (1976).

27. A.Ekimov, A. Onushchenko and A. Efros, Sized quantization of an energy distribution of electrons in microchips of semiconductors // Pis'ma Zh. Eksp. Fiz., A43(6), pp. 337-339, (1986) (in Russian).

28. S.I. Pokutnyi, Spectrum of the exciton in quasi-zero-dimensional semiconductors structures // Fiz. Tekhn. Polupr, 34(11), pp. 1952-1959, (1996) (in Russian).

29. S.I. Pokutnyi, Size quantization of excitons in quasi-zero-dimensional semiconductors structures // Phys. Lett., A168(5,6), pp. 433-436, (1992).

30. V.B. Shikin and Yu. P. Monarkha, Dwumiernyie zaryazhenie systemi v helii, Nauka, Moskva, (1989) (in Russian).

31. M.W. Cole, Property of image potential induced surface state of insulators// Phys. Rev., B2(10), 4239-4252, (1970). 